$$
\begin{aligned}
& \text { استخدام مغير مصدر الفولتية لتنظيم فولتية وتردد المولد الحثي } \\
& \text { ثلاثي الطور ذاتي الإثارة } \\
& \text { أ.د. باسل محمد سعيد و السيا. حسن عدنان } \\
& \text { قسم الهندسة الكهربائية } \\
& \text { جامعة الموصل } \\
& \text { الــخلاصــة }
\end{aligned}
$$

تم في هذا البحث عرض وتحليل عمل منظومـة المولد الحثّي ثلاثي الطور ذاتيـة الإثارة في حالـة التشـغيل

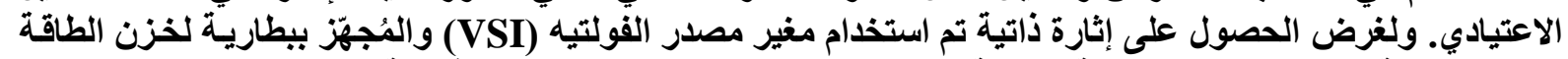

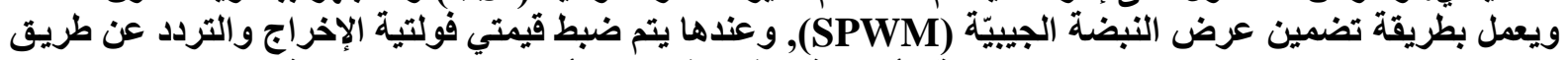

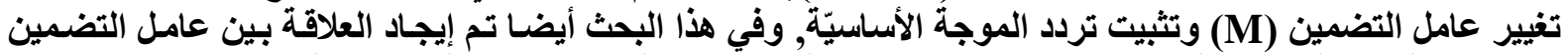

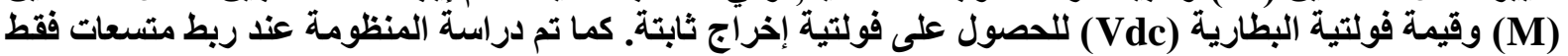

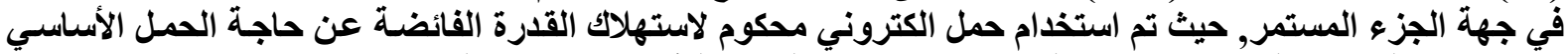

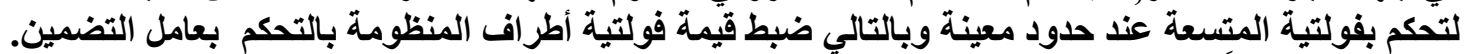

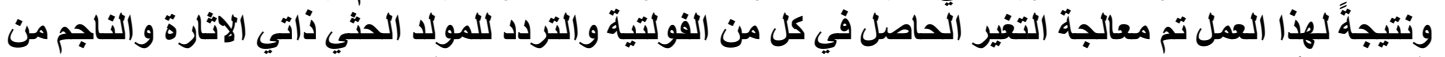

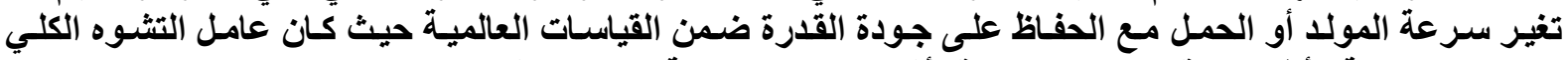
لفولتية الأطر اف اقل من (4HD)

\title{
Voltage and Frequency Regulation of a Three Phase Induction Generator Using Voltage Source Inverter
}

\author{
Prof. Basil M. Saied \\ Electrical Eng. Dept \\ Mosul university
}

Mr. Hasan A.Mohammed

\begin{abstract}
ABSTARCT
In this paper the study of a three phase SEIG system under steady state condition is presented. In order to obtain self excitation, a voltage source inverter (VSI) supported by a battery energy storage system and based on sinusoidal pulse width modulation (SPWM) strategy is used. The output generated voltage and frequency are adjusted by changing modulation index $(M)$. The relationship between the modulation index (M) and (dc) battery voltage source has also been found in order to regulate the value of terminal voltage. This system has been also examined with the existence of capacitor located at the D.C link side of the inverter, electronic load has been used to absorb the excess power and also to limit the DC voltage across the capacitor from exceeding the limit, while the generated terminal voltage is fixed by adjusting the modulation index.

As a result the terminal voltage and frequency fluctuation due to load and speed changes are eliminated. The generated voltage waveform is sinusoidal with (THD) less than $(4 \%)$, keeping power quality within the international standards. both simulation and experimental results are in agreement with objectives of this research.
\end{abstract}

$$
28 \text { - قبل: } 2011 \text { - } 4
$$$$
29 \text { أستلم: } 2011 \text { - } 20
$$ 


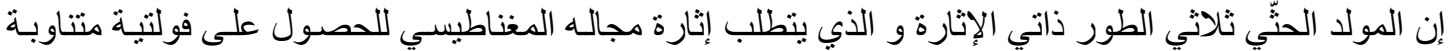

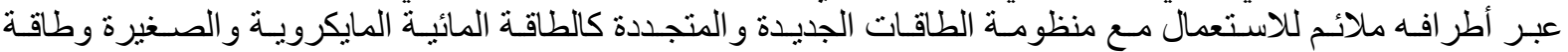

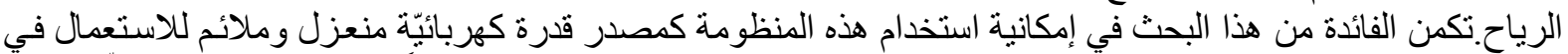

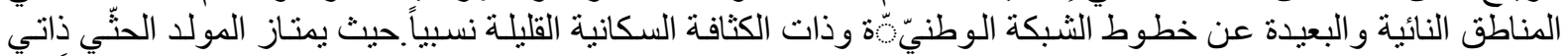

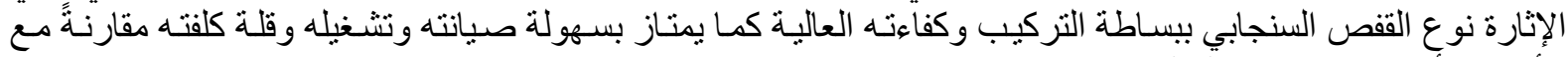

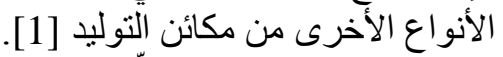

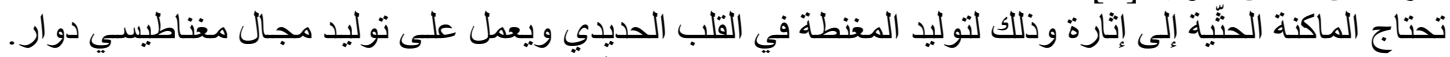

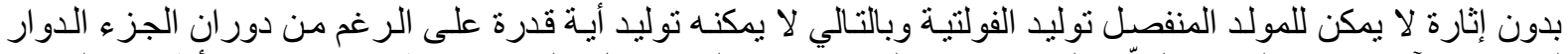

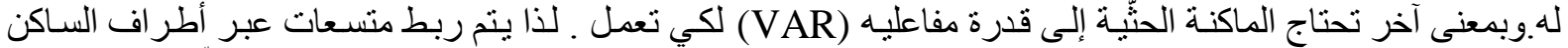

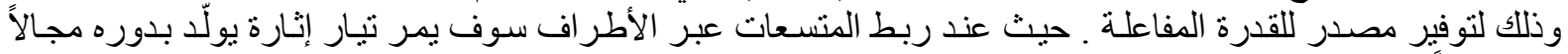

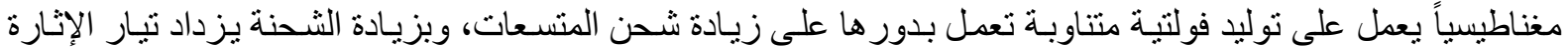

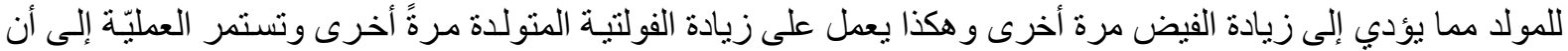

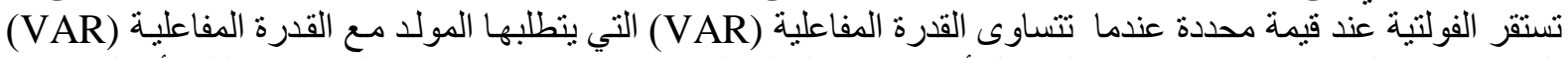

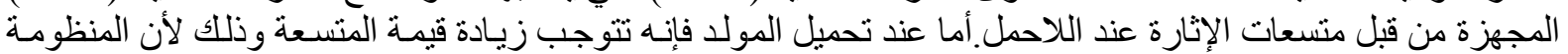

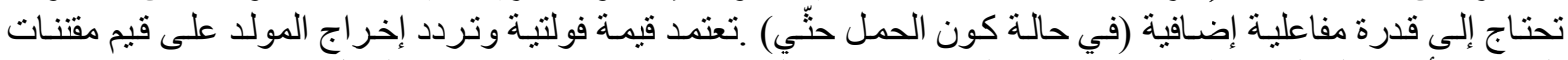

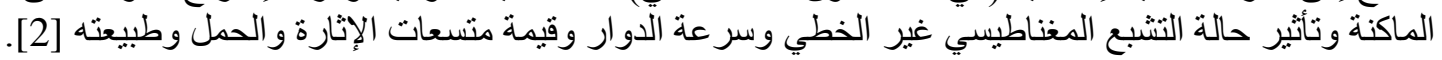

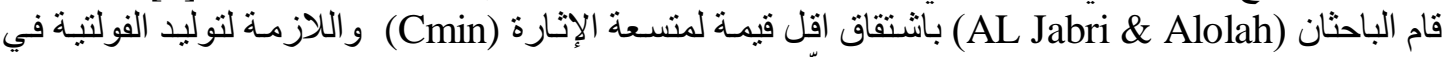

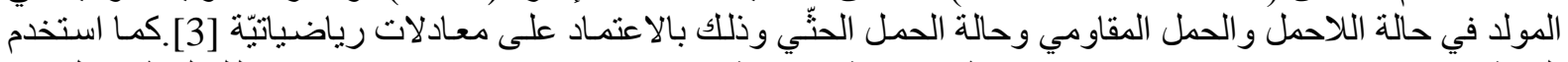

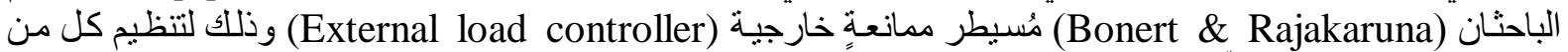

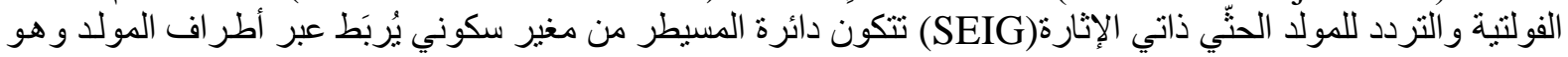

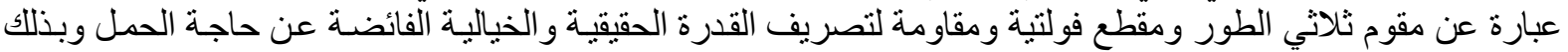

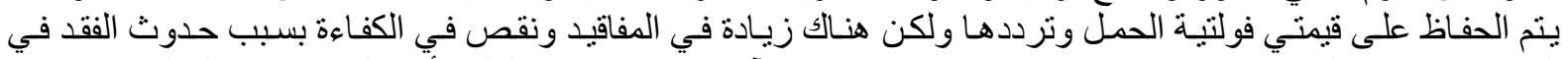

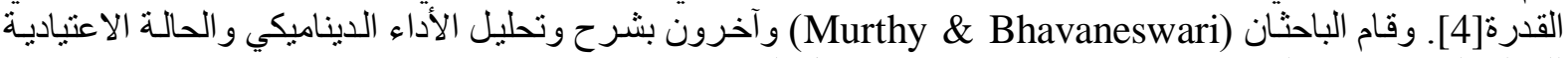

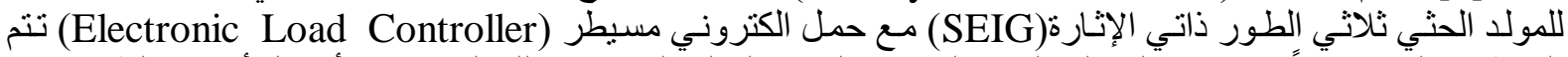

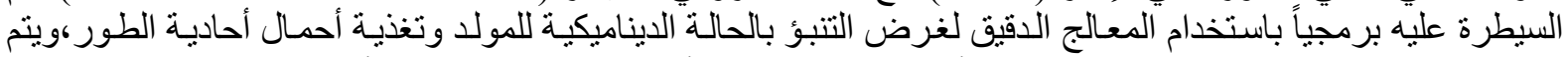

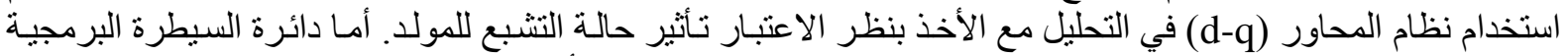

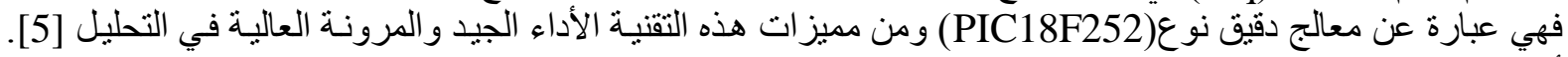

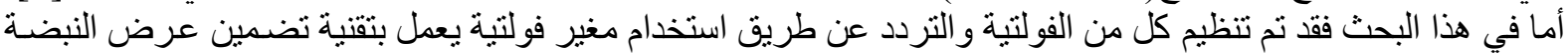

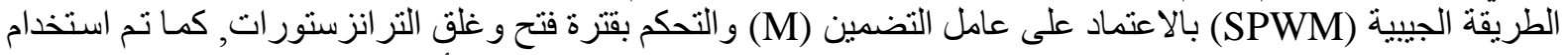

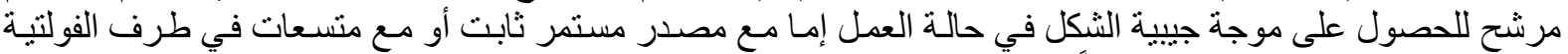
المستمرة لمغير مصدر الفولنية مضافاً إليها حمل الكترونية العمل مسيطر.

\section{2: 20 منظومة المولا الحنّي ثلاثي الطور ذاتية الإثارة.}

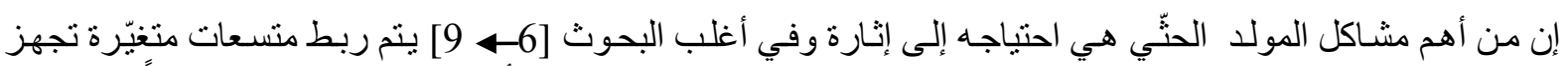

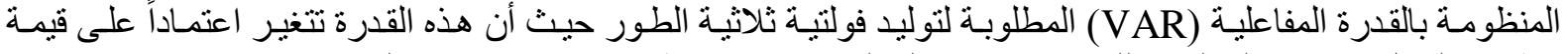

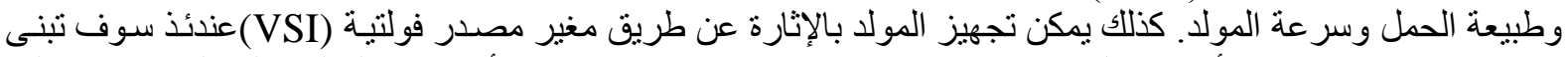

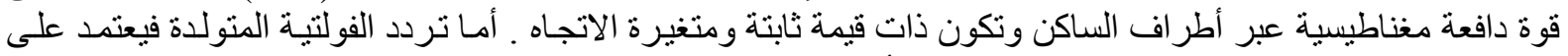

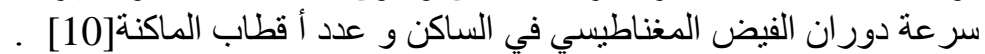

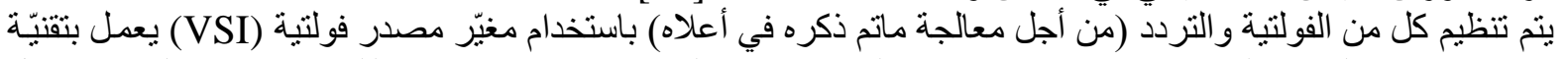

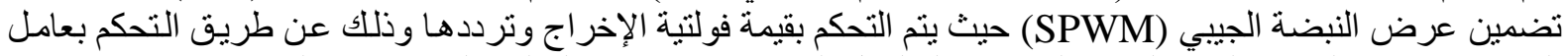

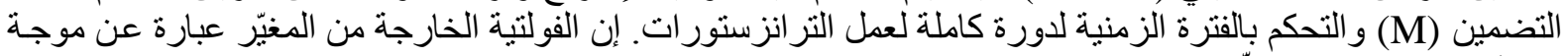

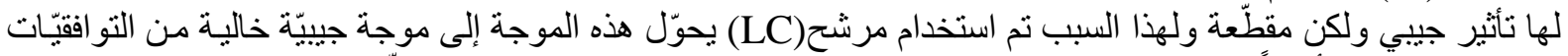

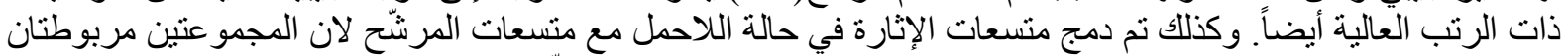

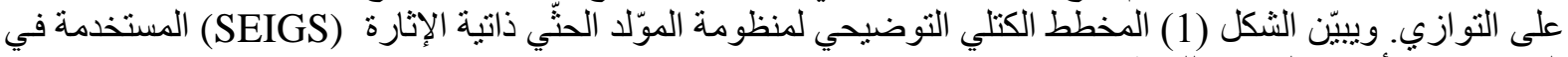
البحث ـ إن ألأجز اء الرئيسة للمنظومة هي: 
سعيد: استخدام مغير مصدر الفولتية لتظظيم فولتية وتردد المولد الحثي ثلاثي الطور ذاتي الإثارة

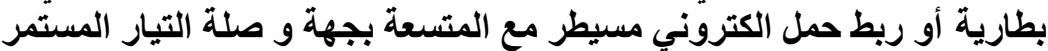
• مرشح (LC) ثلاثي الطور لترشيح التوافقيات ذات الرتب العالية مضافة إلى متسعة الإثارة

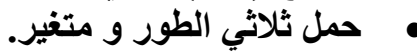

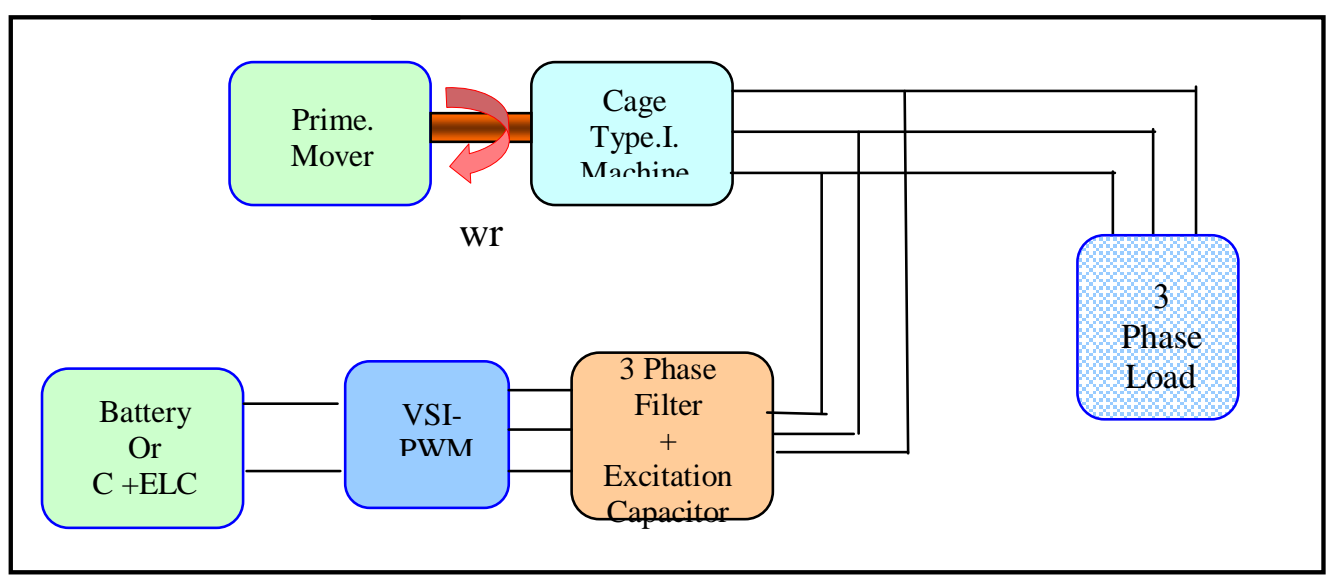

الشكل (1) المخطط الكتلي لمنظومة المولد الحثي ثلاثي الطور ذاتية

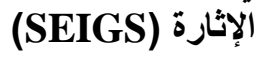

كما وييّين الثكل (2) تركيب المنظومة موّضحاً جميع العناصر الرئيسة و المستخدمة في تنظيم كل من الفولتية

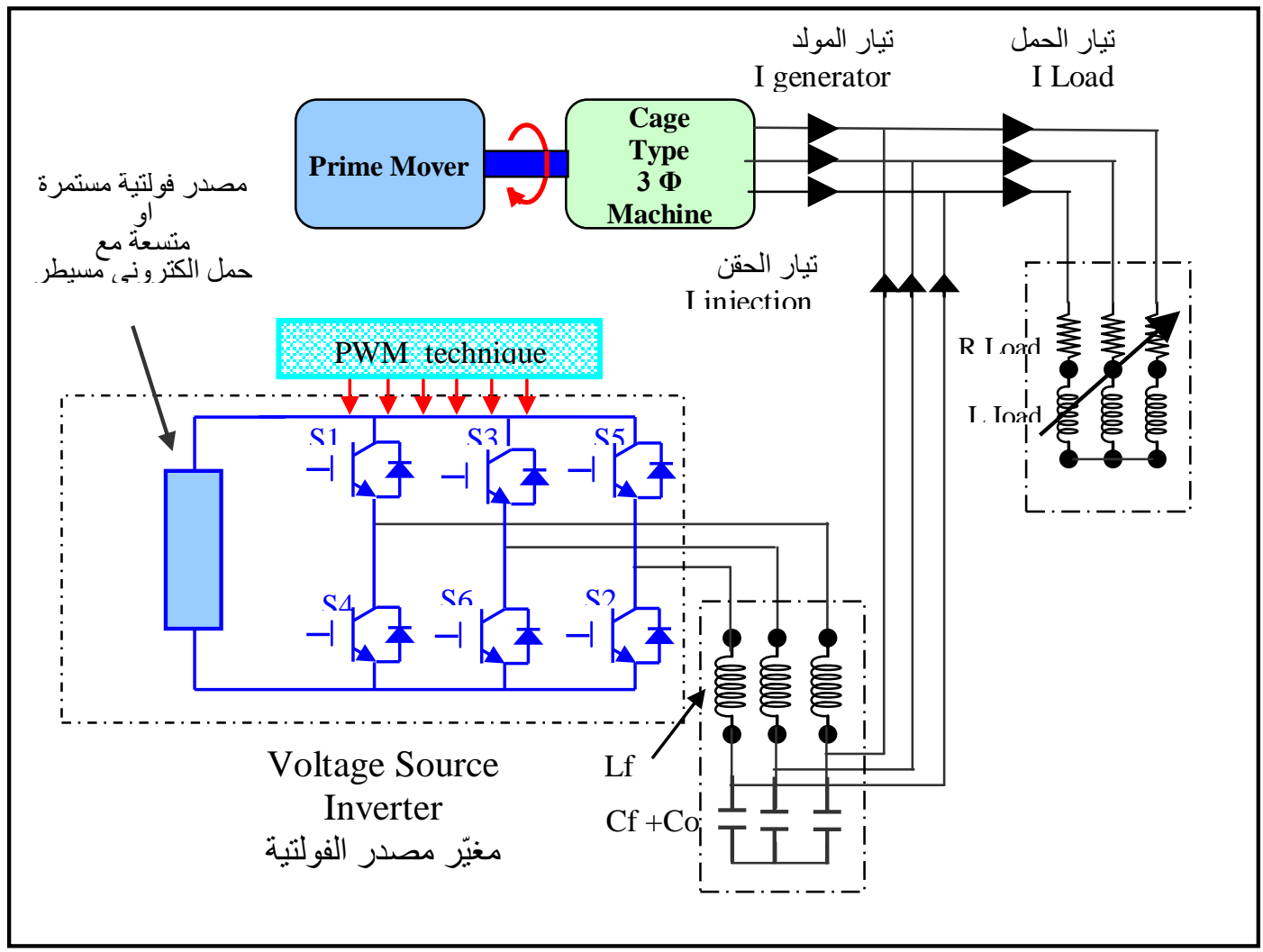

الثكل (2) منظومة المولا الحَّي ثُلاثي الطور ذاتية الإثارة

.(SEIGS) 


\section{1-2 1-2 مغير مصدر القولتية ثلاثي الطور (VSI).}

إن التركيب الأساس لمغيّر مصدر الفولتيّة (VSI) مبيّن بالثكل (2), حيث أن هذا النوع من المئ المغيّر قد تم

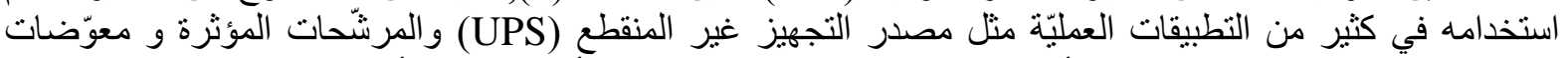

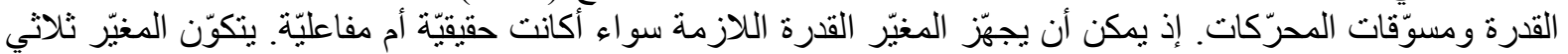

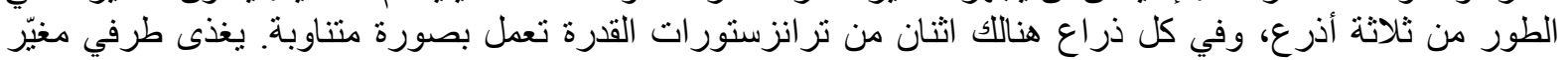

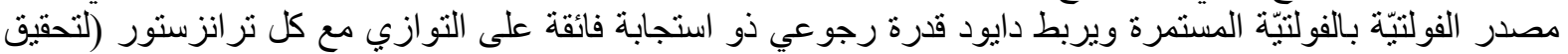

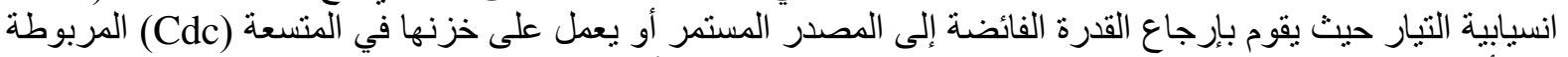

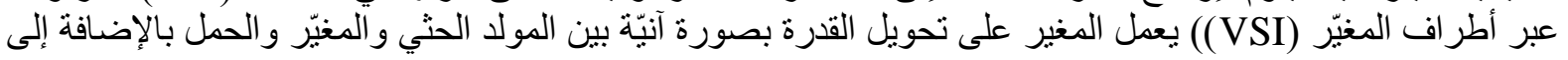

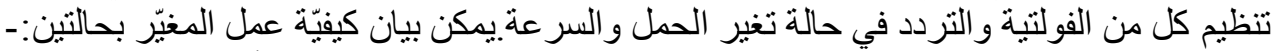

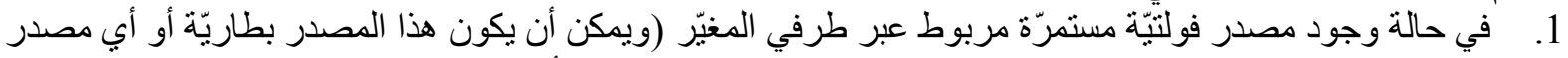

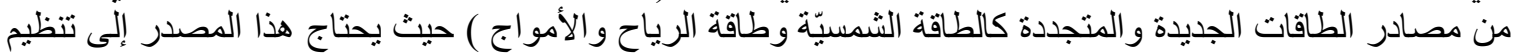

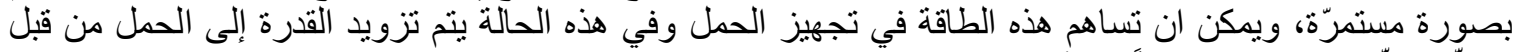

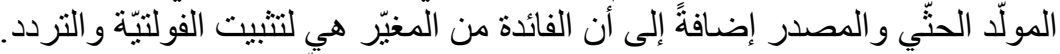

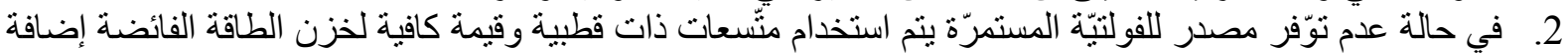

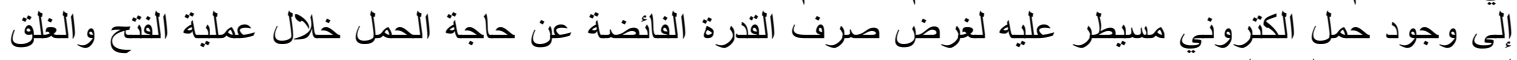
لتجنب زيادة فولتية المتسعة.

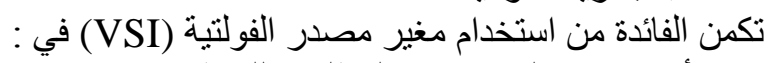

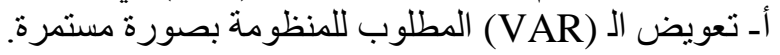

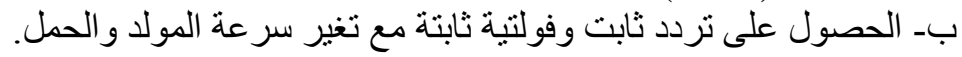

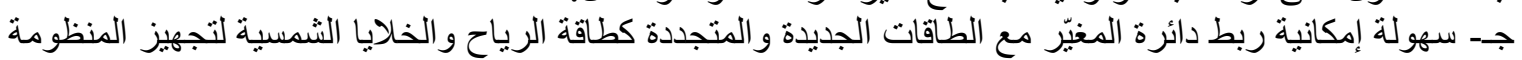

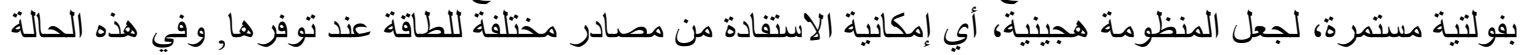

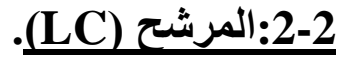

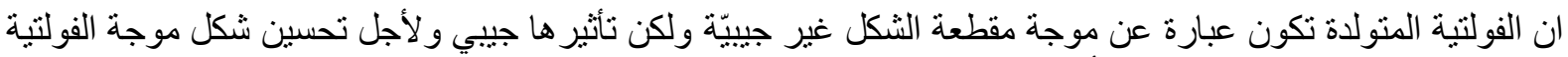

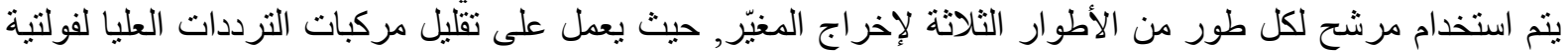

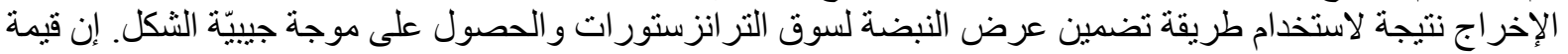
تموّج التيار الذي يمر في الملف يعتمد على قيمة محاثة الملف (1)

$$
L_{f}=\frac{V d c}{4 f_{c} \Delta i}
$$

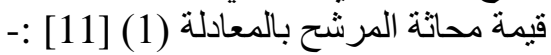

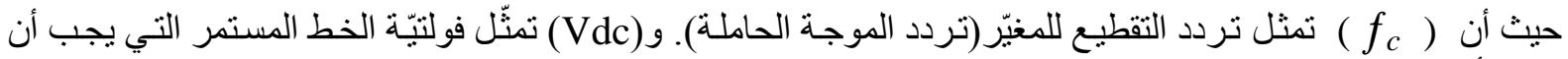

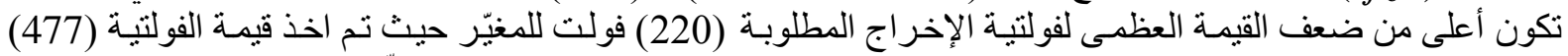

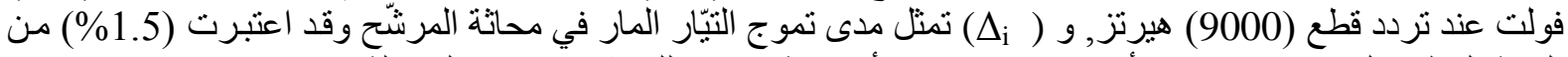
القيمة العظمى لتيار الإخر اج وقد أخذت (

$$
C_{f}=\frac{\Delta i}{8 f \Delta V_{0}}
$$

$\Delta V_{0}$

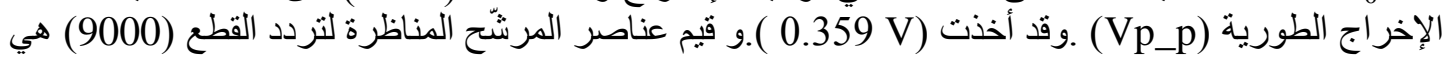

$$
\left(L_{f}=4 m H \& C_{f}=125 \mu f\right)
$$


سعيد: استخدام مغير مصدر الفولتية لتظيم فولتية وتردد المولا الحثي ثلاثي الطور ذاتي الإثارة

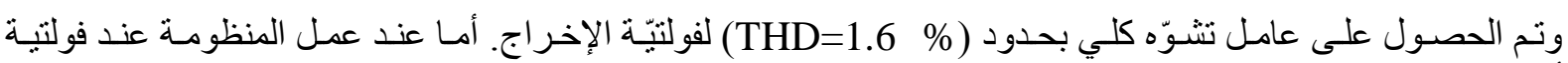
أطر اف(380) فولت فان عان

$$
\left(L_{f}=5.5 m H \& C_{f}=80 \mu f\right)
$$

المناظرة للمنظومة ونم الحصول على عامل تشوّه كلي (\% \%

\section{3-2 طبيعة الحمل المستخدم مع الموّلد الحثّي.}

يعمل المولد بتجهيز القدرة لحمل ثلاثي الطور وقد تم استخدام حمل حثي ذي عامل قدرة متغير.

\section{3: الطريقة الجيبية لتضمين عرض النبضة (SPWM).}

لغرض تحسين شكل الفولتية وتنظيم قيمتها يتم استخدام تقنية تضمين عرض النبضة الجيبي (SPWM) لسوق دائرة مغيّر

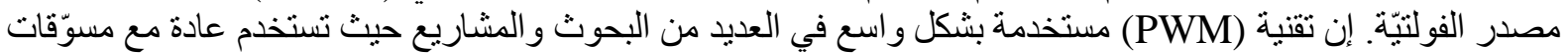

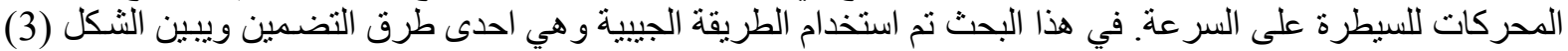

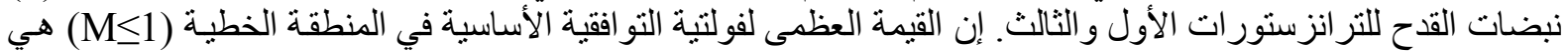

$$
\text { ( vab = } \sqrt{3} \frac{V d c}{2}\left(\frac{V d c}{2}\right)
$$

لذاللك يمكن كتابة القيمة العظى لفولتية الإخر اج الخطية بالمعادلة (3) الآتية [12] .

$\hat{v} a b=M \sqrt{3} \frac{V d c}{2} \quad$ for $\quad 0<M \leq 1$.

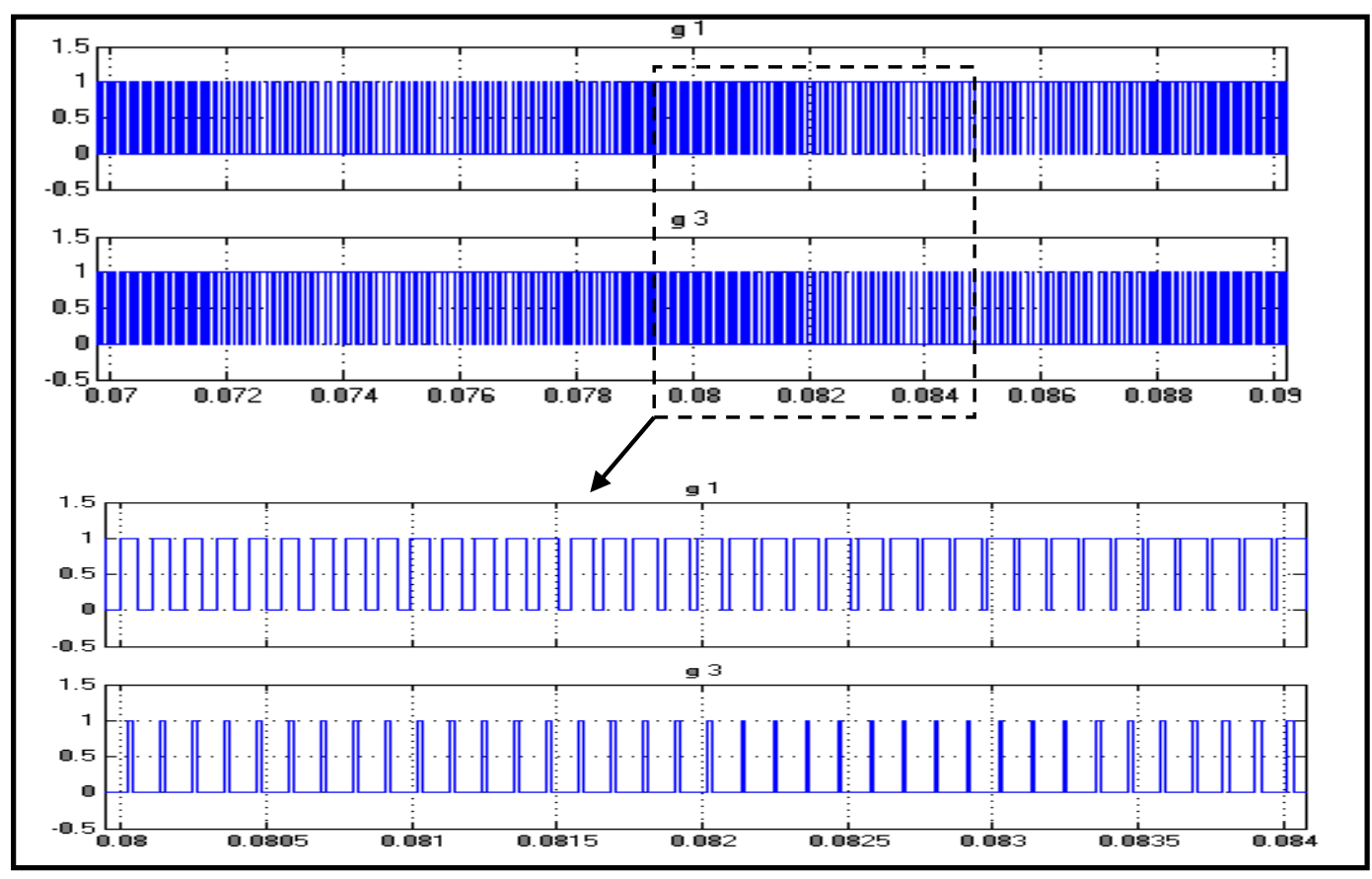

الثكل (3) نبضات القدح للترانزستورات الأول والثالث

4: 1: نتائج التمثيل الحاسوبي.

تم عرض نتائج التمثيل الحاسوبي للمنظومة باستخدام نظام (Matlab 7.4). تم دمج متسعات الإثارة مع متسعات المرشح

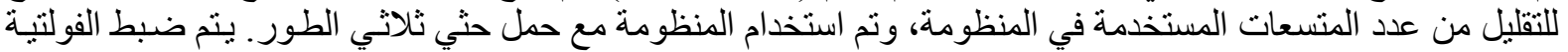

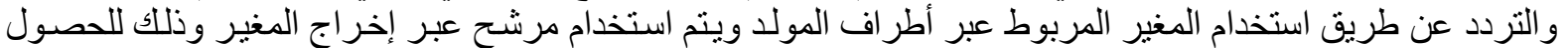

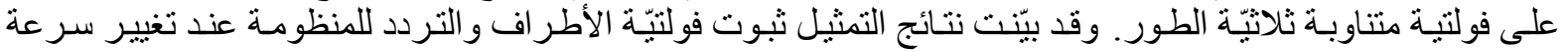


المحرّك البادئ وكذلك عند استخدام أحمال حتّيّة متغيّرة أما بالنسبة لخط الفولتيّة المستمرة فقد تم تقسيم حالات العمل إلى

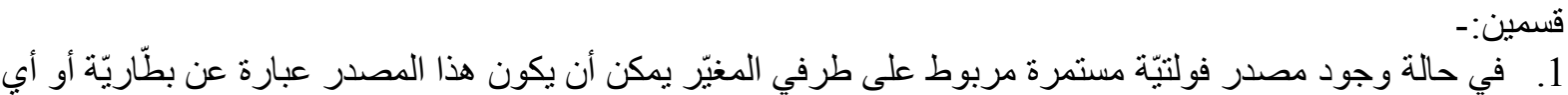

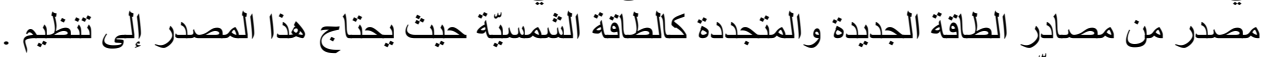

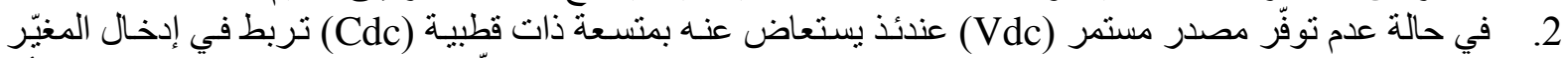

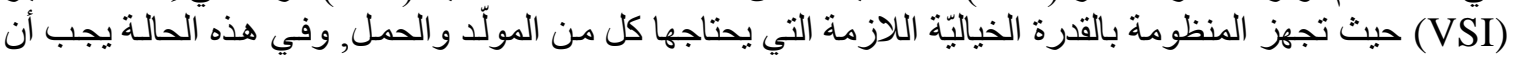

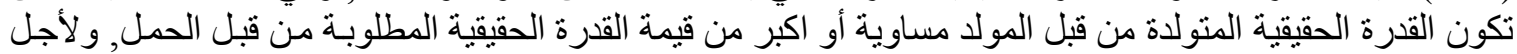

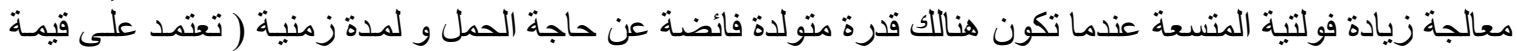

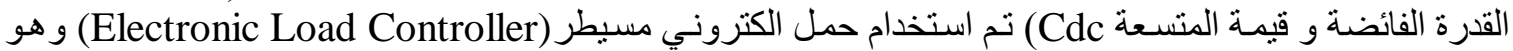

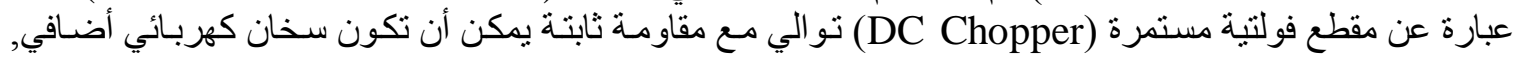

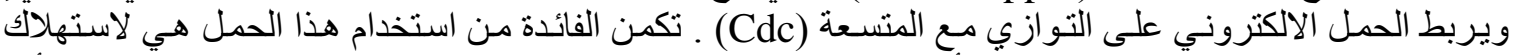

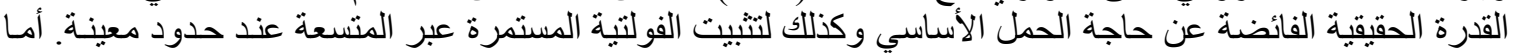

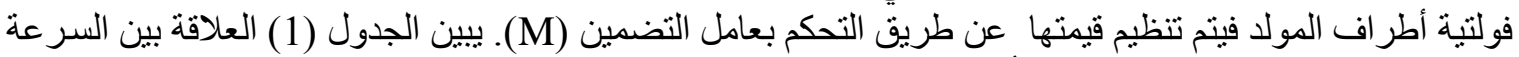

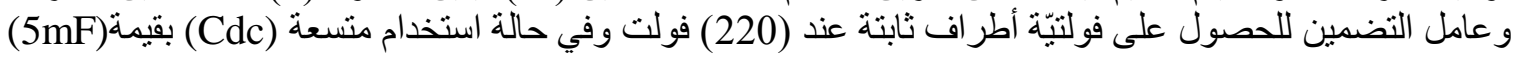

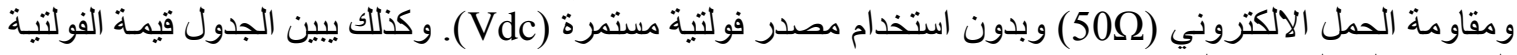
المستمرة المتولدة عبر المتسعة.

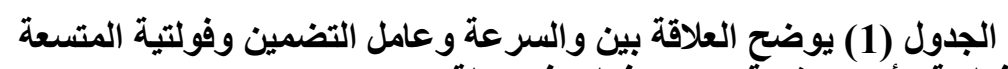

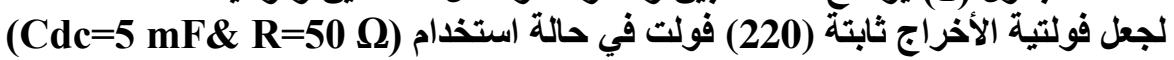

\begin{tabular}{|c|c|c|c|c|c|c|c|c|c|c|}
\hline N(R.P.M) & $\mathbf{1 5 6 0}$ & $\mathbf{1 5 7 0}$ & $\mathbf{1 5 8 0}$ & $\mathbf{1 5 9 0}$ & $\mathbf{1 6 0 0}$ & $\mathbf{1 6 1 0}$ & $\mathbf{1 6 2 0}$ & $\mathbf{1 6 3 0}$ & $\mathbf{1 6 4 0}$ & $\mathbf{1 6 5 0}$ \\
\hline $\mathrm{M}$ & 0.839 & 0.827 & 0.818 & 0.808 & 0.8 & 0.793 & 0.787 & 0.781 & 0.779 & 0.777 \\
\hline $\mathrm{Vdc}$ & 448.8 & 456.5 & 463.5 & 470.8 & 477 & 482.7 & 488.3 & 493.4 & 498.4 & 503.2 \\
\hline
\end{tabular}

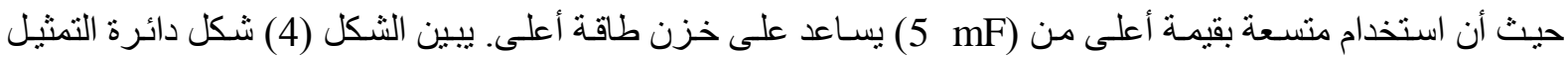

الحاسوبي للحمل الالكتروني المسيطر (ELC).

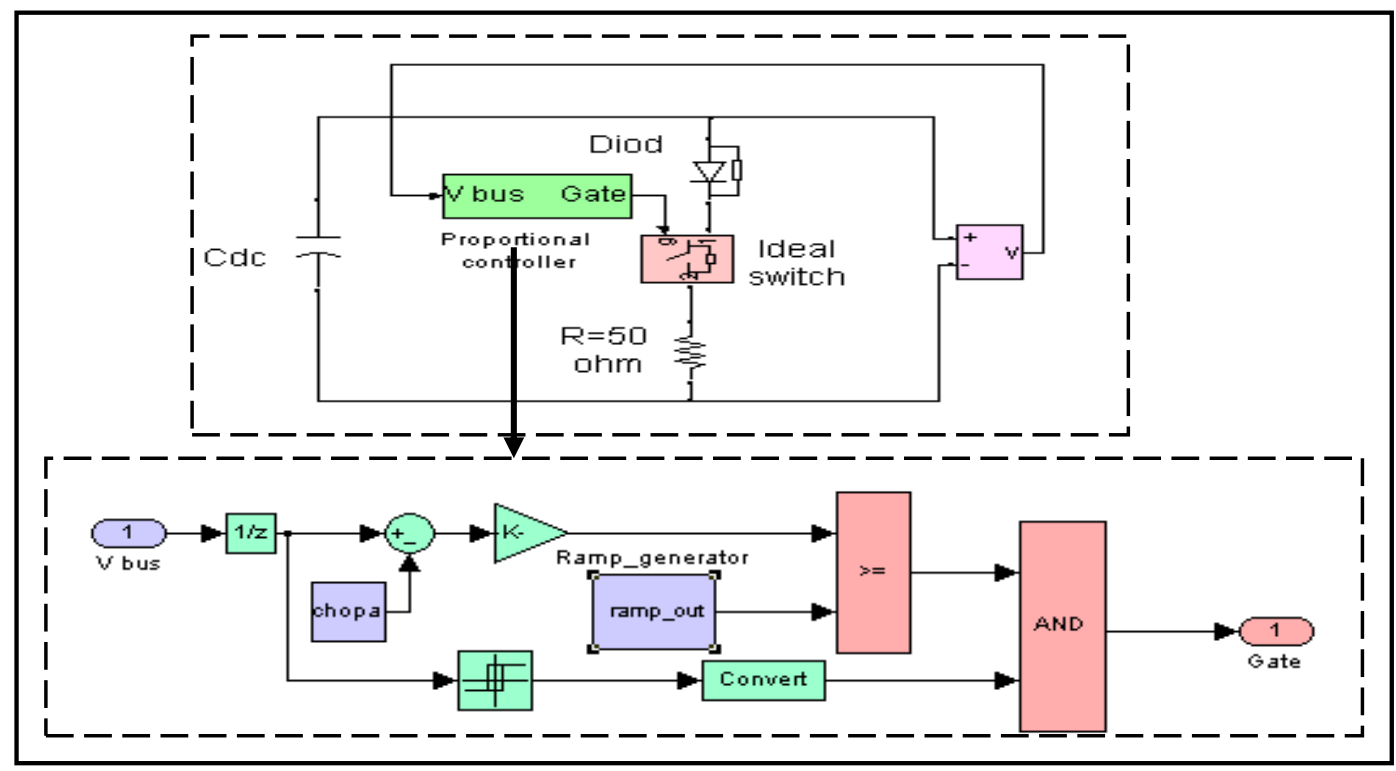

الثكل (4)دائرة التمثيل الحاسوبي للحمل الاكتروني المسيطر (ELC). 


\section{1-5}

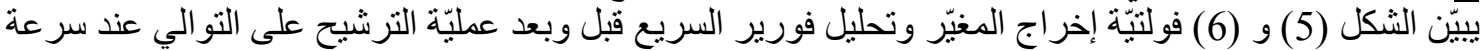

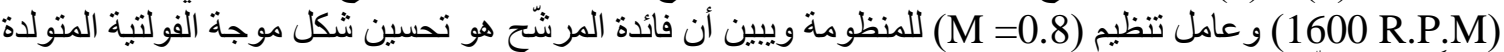

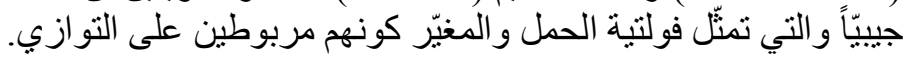

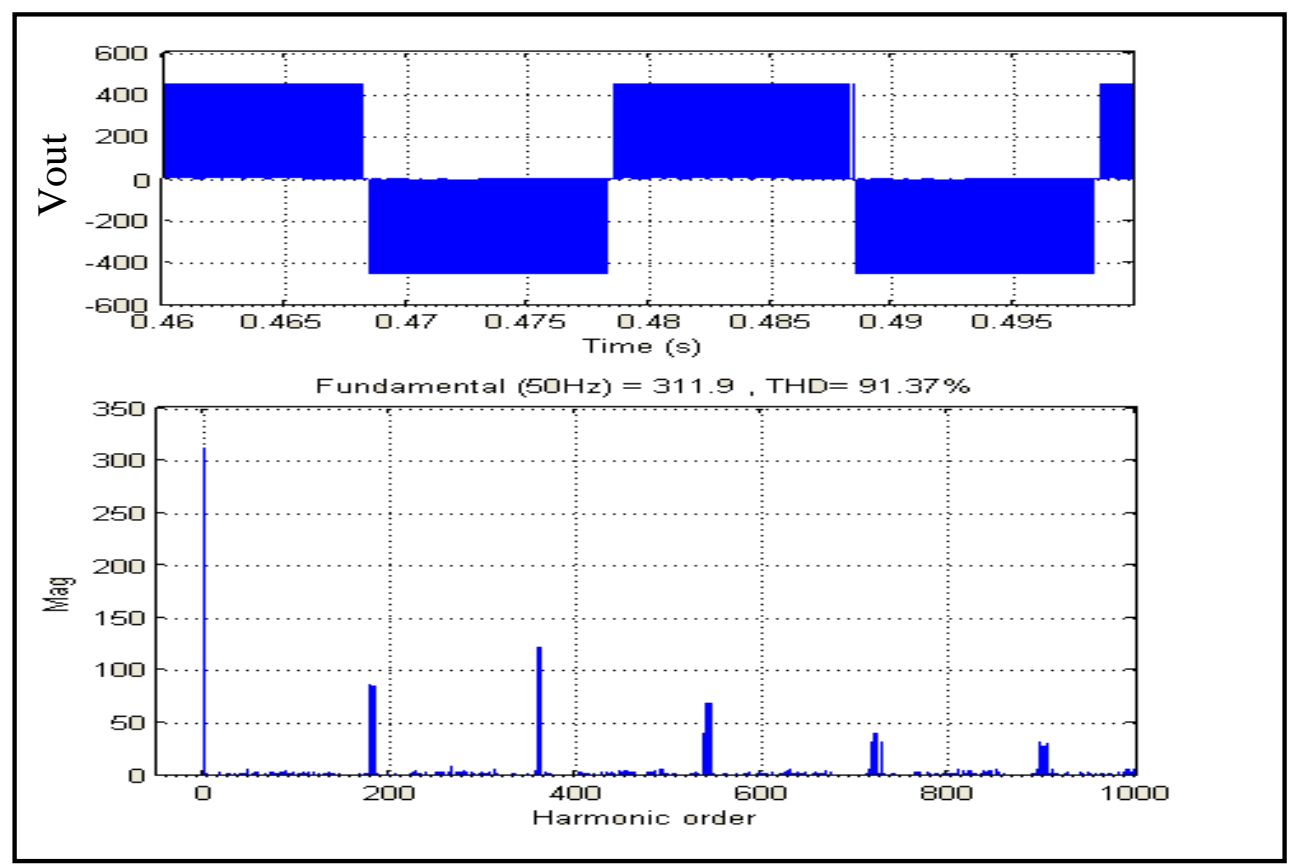

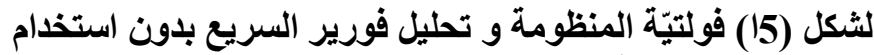

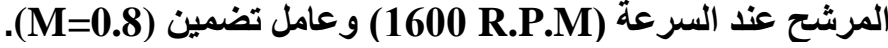

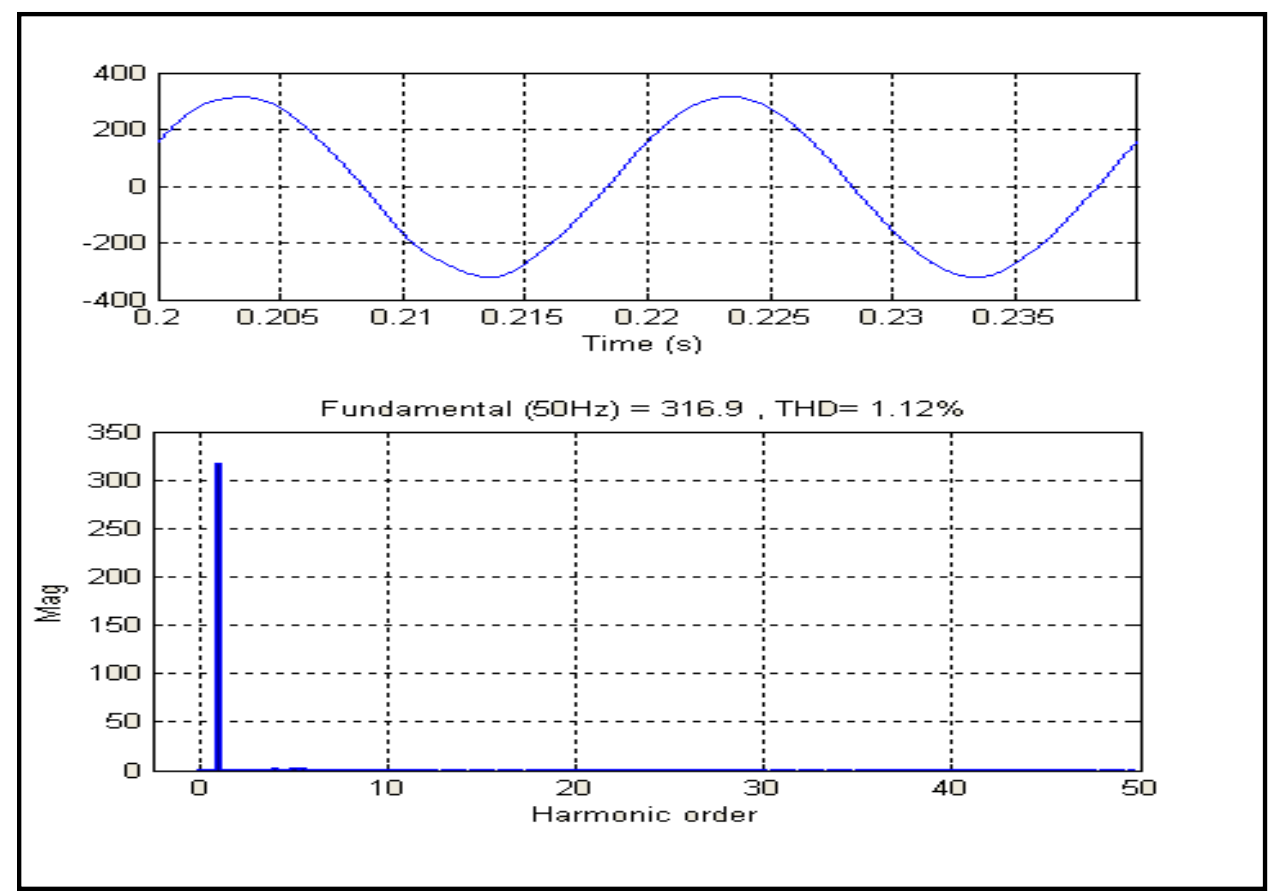

الثكل (6) فولتيّة المنظومة و تحليل فورير السريع بعد استخدام

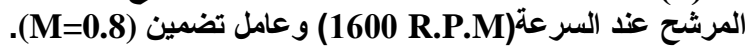




\section{2-5: نتائج التمثيل الحاسوبي لفولتيات وتيارات المنظومة.}

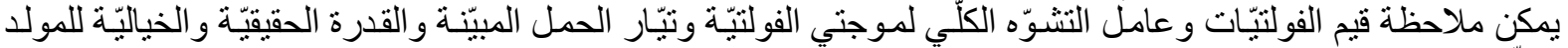

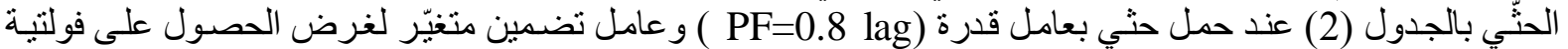

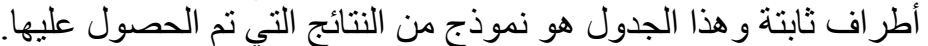
ملاحظة:-تم أخذ نتائج التمثيل في حالة عمل المنظومة عندة عند فولتية خط (220) فولت بتردد (50) هيرتز ومصدر فولتيـة

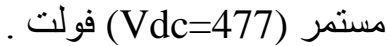

الجدول (2) يوضح علاقة فولتية المولد و عامل التشوه الكلي للفولتية والقدرة الحقيقية والخياليّة للمولا عند سرع مختلفة وتردد قطع (fc=9 KHz) وعامل تضمين متغيّر

\begin{tabular}{|c|c|c|c|c|c|c|c|c|c|c|}
\hline $\begin{array}{c}\mathrm{N} \\
(\text { R.P.M })\end{array}$ & $\mathrm{M}$ & $\begin{array}{c}\text { Vout } \\
\text { (Volt) }\end{array}$ & $\begin{array}{c}\text { THD\% } \\
\text { For } \\
(\mathrm{V})\end{array}$ & $\begin{array}{c}\text { THD\% } \\
\text { For } \\
\text { (Iload) }\end{array}$ & $\begin{array}{c}\text { Pg/Ph } \\
\text { (Watt) }\end{array}$ & $\begin{array}{c}\text { Qg/ph } \\
\text { (VAR) }\end{array}$ & $\begin{array}{c}\text { Pinj/Ph } \\
\text { (Watt) }\end{array}$ & $\begin{array}{c}\text { Qinj/ph } \\
\text { (VAR) }\end{array}$ & $\begin{array}{c}\text { Pload/Ph } \\
\text { (Watt) }\end{array}$ & $\begin{array}{c}\text { Qload/ph } \\
\text { (VAR) }\end{array}$ \\
\hline 1500 & 0.782 & 220 & 1.6 & 0.6 & -31.96 & -708.8 & 549 & -708.8 & 517 & 387.5 \\
\hline 1510 & 0.782 & 220.3 & 1.9 & 0.6 & 72.91 & -720.4 & 443.4 & -720.4 & 517 & 387.5 \\
\hline 1520 & 0.782 & 220 & 1.9 & 0.6 & 178.4 & -735.3 & 336.9 & -735.3 & 517 & 387.5 \\
\hline 1530 & 0.783 & 220.1 & 1.6 & 0.6 & 279.8 & -754.9 & 235.7 & -754.9 & 517 & 387.5 \\
\hline 1540 & 0.784 & 220.2 & 1.4 & 0.5 & 384 & -782.8 & 131.9 & -782.8 & 517 & 387.5 \\
\hline 1550 & 0.786 & 220 & 1 & 0.4 & 492.4 & -828.4 & 26.1 & -828.4 & 517 & 387.5 \\
\hline 1560 & 0.787 & 219.9 & 1.3 & 0.3 & 595.4 & -854.7 & -78.26 & -854.7 & 517 & 387.5 \\
\hline 1570 & 0.79 & 220.2 & 1.3 & 0.4 & 708.8 & -891.6 & -191.5 & -891.6 & 517 & 387.5 \\
\hline 1580 & 0.792 & 219.8 & 1.2 & 0.4 & 814.5 & -929.3 & -298.5 & -929.3 & 517 & 387.5 \\
\hline 1590 & 0.796 & 220.1 & 1.4 & 0.4 & 927.7 & -977.8 & -411.1 & -977.8 & 517 & 387.5 \\
\hline 1600 & 0.8 & 220.1 & 1.6 & 0.6 & 1028 & -1026 & -511.7 & -1026 & 517 & 387.5 \\
\hline 1610 & 0.804 & 220 & 1.6 & 0.5 & 1130 & -1084 & -614.1 & -1084 & 517 & 387.5 \\
\hline 1620 & 0.809 & 220 & 2 & 0.5 & 1233 & -1153 & -715 & -1153 & 517 & 387.5 \\
\hline 1630 & 0.813 & 220 & 2.1 & 0.7 & 1342 & -1222 & -824.4 & -1222 & 517 & 387.5 \\
\hline 1640 & 0.818 & 220 & 1.3 & 0.5 & 1441 & -1281 & -924.5 & -12811 & 517 & 387.5 \\
\hline 1650 & 0.821 & 220.1 & 1.6 & 0.6 & 1533 & -1358 & -1016 & -1358 & 517 & 387.5 \\
\hline
\end{tabular}

3-5: تغير انسيابيّة القدرة الحقيقية والخيالية لعناصر المنظومة.

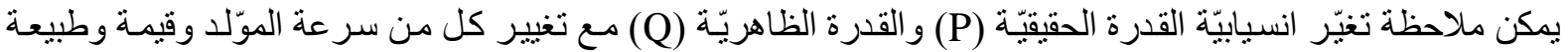

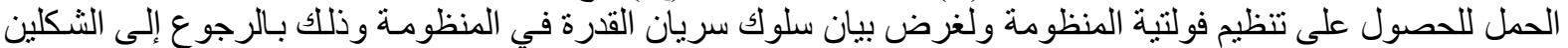

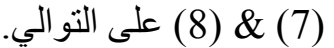

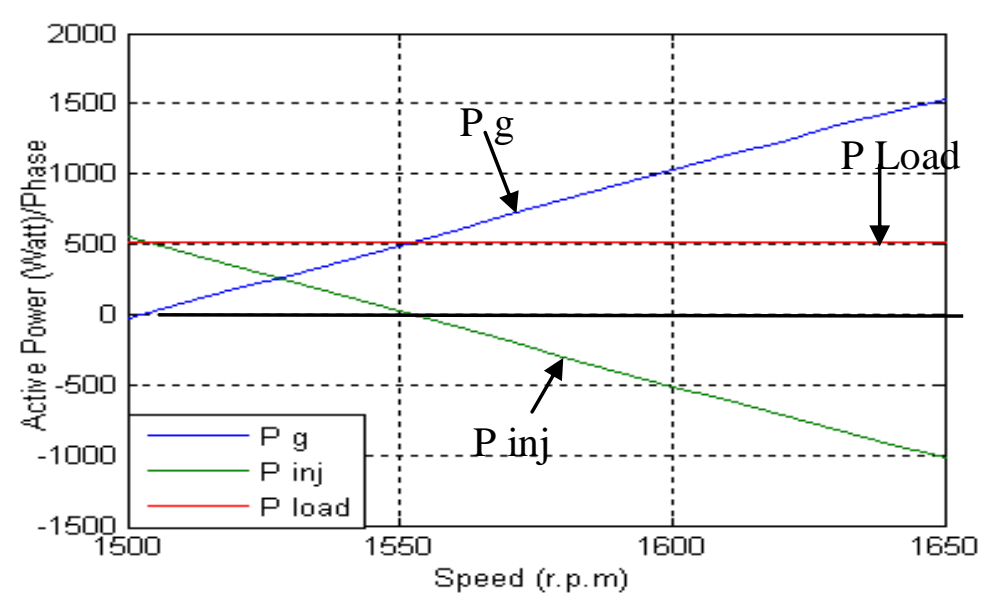

شكل (7) مخطط تغيّر القدرة الحقيقّة لكل طور للمنظومة مع السرعة للمنظومة 
سعيد: استخدام مغير مصدر الفولتية لتظيم فولتية وتردد المولا الحثي ثلاثي الطور ذاتي الإثارة

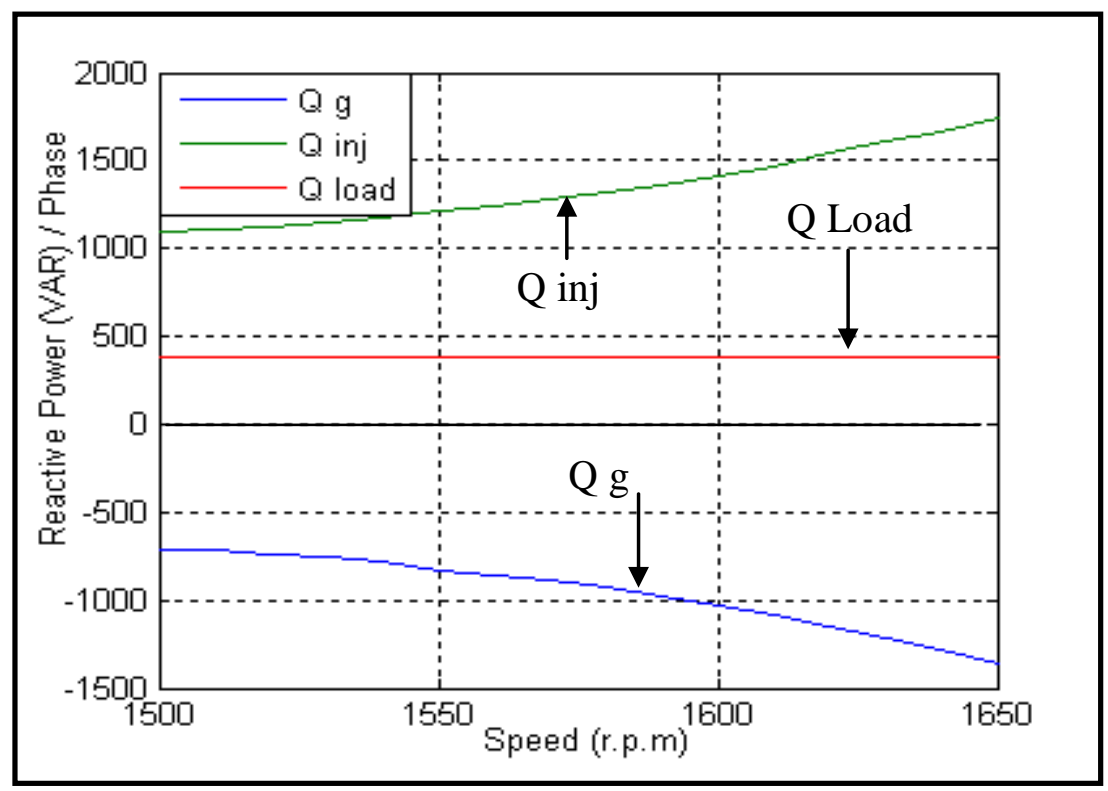

شكل (8) مخطط تغيّر القدرة الخيالية لكل طور للمنظومة مع السرعة للمنظومة

4-5 : نتائج التمثيل الحاسوبي للمنظومة فئ عند أحمال مختلفة.

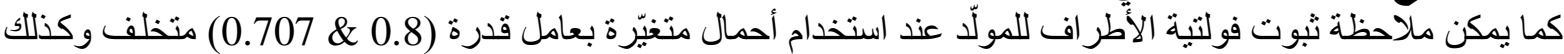
لعامل قدرة واحد ولمنظومة (220) فولت في الجداول (3),(4) و (5) على التو الي في حالة وجود مصدر فولتئة مستمرة . $(\mathrm{Vdc}=477 \mathrm{~V})$

الجدول (3) علاقة فولتيّة ألإخراج و عامل التثّوه للفولتية و القدرة مع الحمل بعامل قرة (0.8) وسرعة (1600 R.P.M)

\begin{tabular}{|c|c|c|c|c|}
\hline Load(0.8pf) & M & Vout (volt) & THD\% & P load/Phase(watt) \\
\hline $20 \Omega \& 47.74 \mathrm{mH}$ & $\mathbf{0 . 8}$ & $\mathbf{2 2 0 . 1}$ & 1.6 & 515.9 \\
\hline $50 \Omega \& 119.35 \mathrm{mH}$ & $\mathbf{0 . 7 8 6}$ & 219.9 & 1.1 & 207.2 \\
\hline $75 \Omega \& 179.025 \mathrm{mH}$ & $\mathbf{0 . 7 8 3}$ & 220 & 1.8 & 137.3 \\
\hline $100 \Omega \& 238.7 \mathrm{mH}$ & 0.782 & 220 & 2.3 & 103 \\
\hline
\end{tabular}

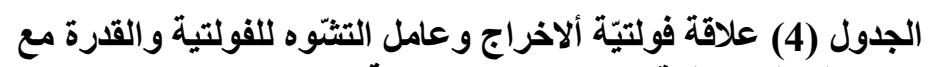

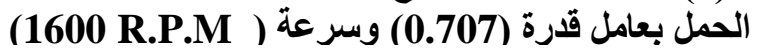

\begin{tabular}{|c|c|c|c|c|}
\hline Load(0.707pf) & M & Vout (volt) & THD\% & P load /Phase(watt) \\
\hline $20 \Omega \& 63.6 \mathrm{mH}$ & $\mathbf{0 . 8}$ & $\mathbf{2 1 9 . 8}$ & 1.5 & $\mathbf{4 0 2}$ \\
\hline $50 \Omega \& 159.1 \mathrm{mH}$ & $\mathbf{0 . 7 8 7}$ & $\mathbf{2 2 0 . 2}$ & 1.3 & $\mathbf{1 6 2 . 2}$ \\
\hline $75 \Omega \& 238.7 \mathrm{mH}$ & $\mathbf{0 . 7 8 3 5}$ & 220 & 1.9 & 107.3 \\
\hline $100 \Omega \& 318.3 \mathrm{mH}$ & $\mathbf{0 . 7 8 2 5}$ & 220 & 1.6 & $\mathbf{8 0 . 4 5}$ \\
\hline
\end{tabular}

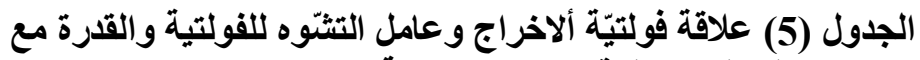

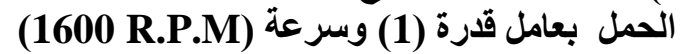

\begin{tabular}{|c|c|c|c|c|}
\hline Load(pf =1) & M & Vout (volt) & THD\% & $\begin{array}{c}\text { Pload/Phase } \\
\text { (watt) }\end{array}$ \\
\hline $20 \Omega$ & 0.774 & 219.9 & 1.4 & 805.7 \\
\hline $50 \Omega$ & 0.7745 & 219.9 & 1.6 & 322.4 \\
\hline $75 \Omega$ & 0.775 & 220.1 & 1.7 & 215.4 \\
\hline $100 \Omega$ & 0.7755 & 220.1 & 1.8 & 161.6 \\
\hline
\end{tabular}




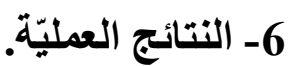

لأجل أثبات عملية التوليد ولأنواع مختلفة من الأحمال وتحسين شكل موجة الفولتية عملياً تم عرض نتائج الجانب

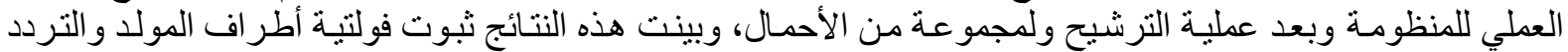

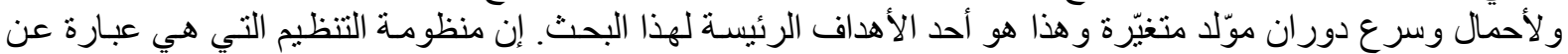

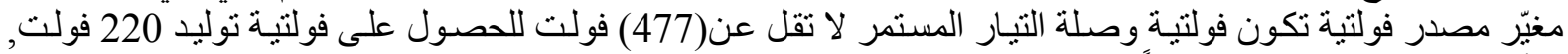

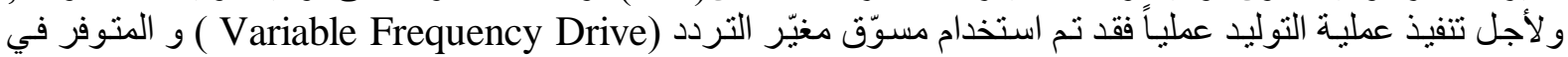

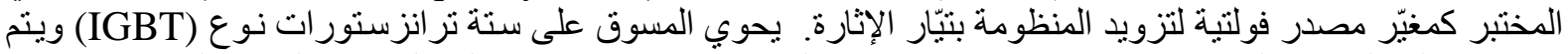

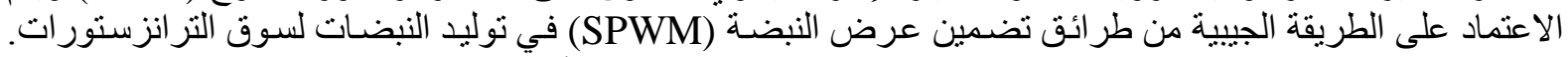

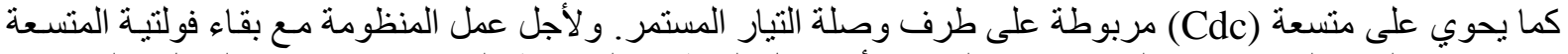

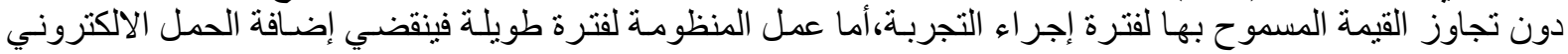

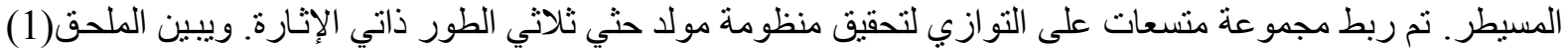
قيم ومقننات المغيّر.

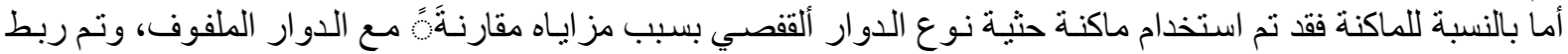

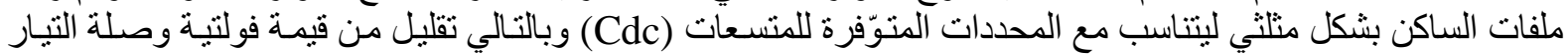

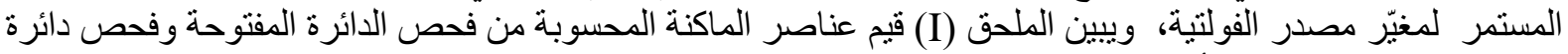

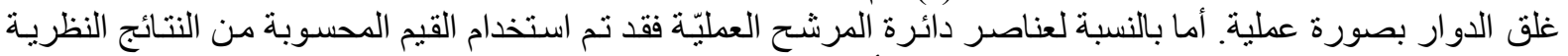

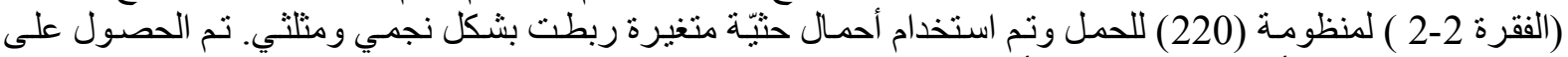

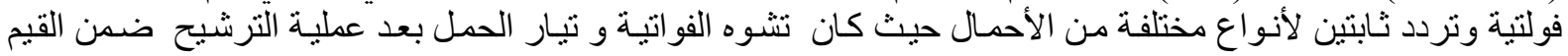

المسموح بها.

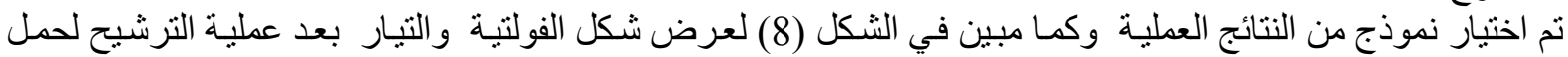

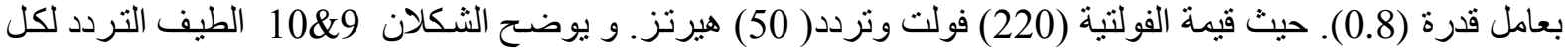
من الفولتية المتولدة عبر الحمل و تيار الحمل بعد إضافة المرشح و كذللك قيمة عامل التشوه الكلي للفولتية و التيار.

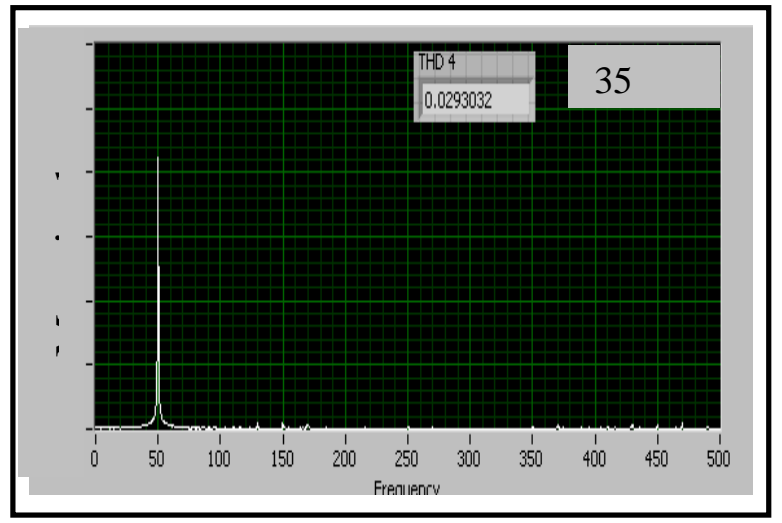

الثكل (9HD \& FFT) (9) لفولتية أطر اف المولا

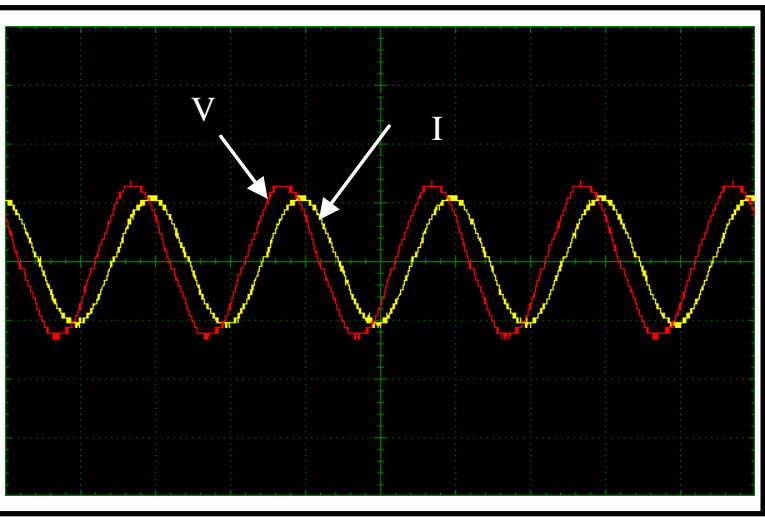

شكل (8) الفولتية والتيار بعد عملية الترشيح لحمل بعامل قدرة 0.8

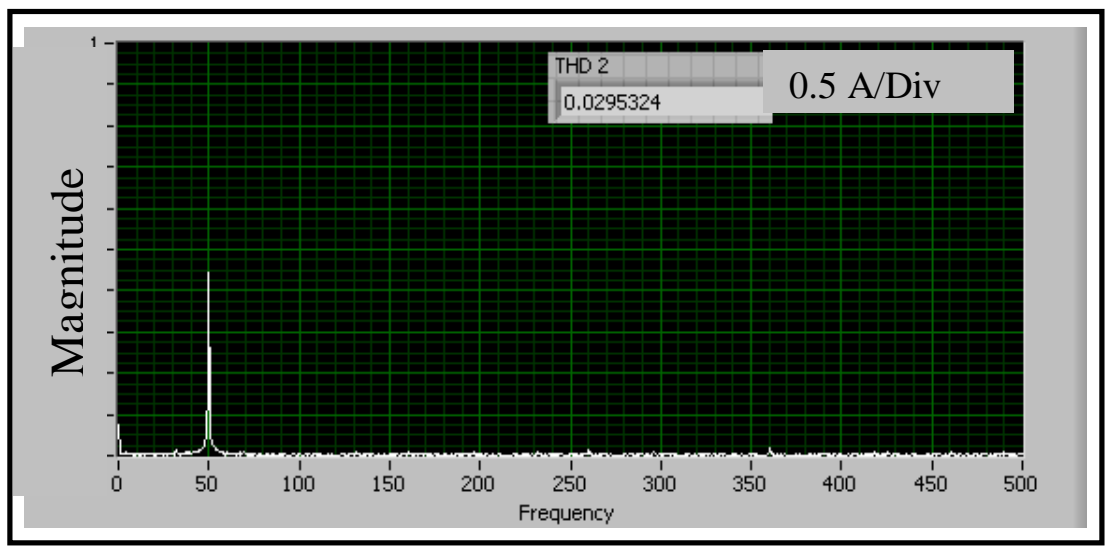

الثكل (10) (THD \& FFT) لتيار الحمل 


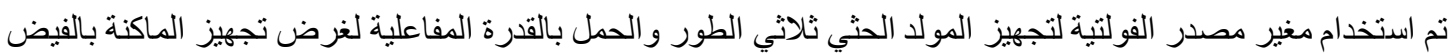

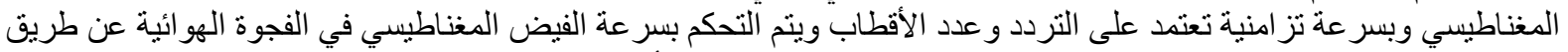

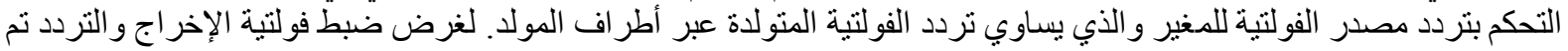

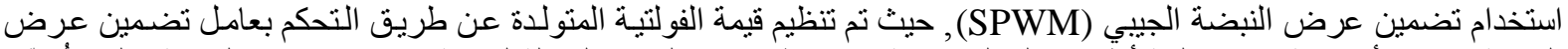

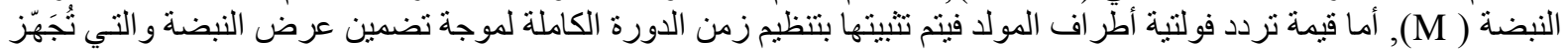

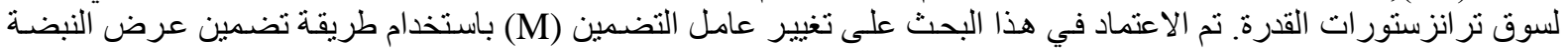

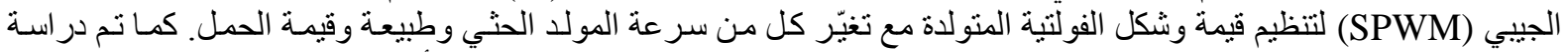

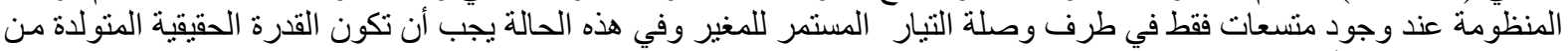

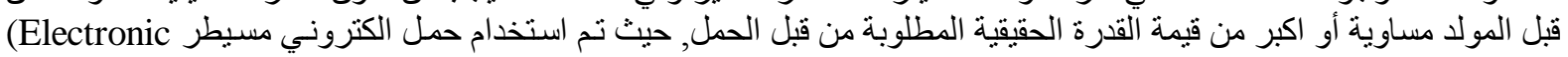

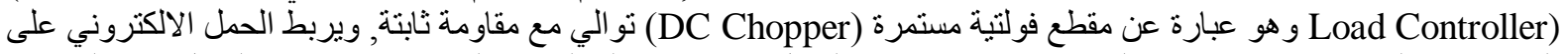

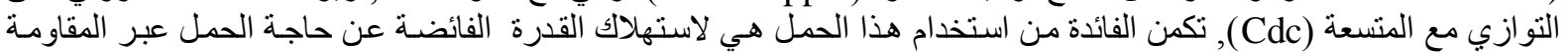

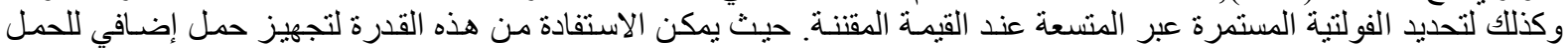
الاساس ( كالسخان الكهربائي مثلا).

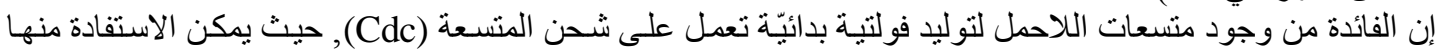

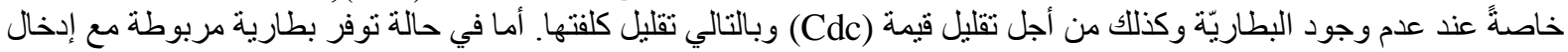

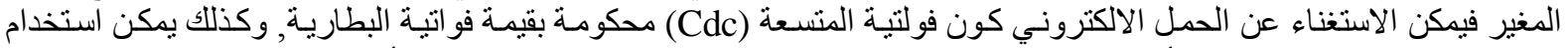

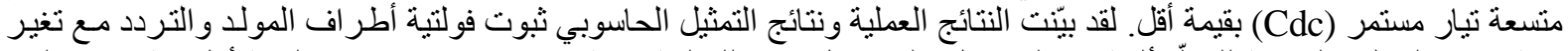

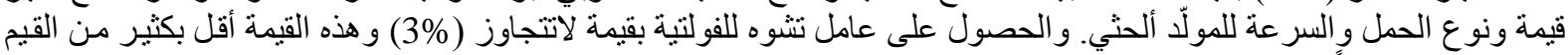

\section{References}

1. N.H .Malik , A.A Mazi "Capacitance Requirements for Isolated Self Excited Induction Generators" IEEE Trans on energy conversion, Vol. EC-2, No.1. March 1987, pp.62 - 69.

2. L.Shridhar, B. Singh, C.S.Jha, and B.P. Singh "Analysis of Self Excited Induction Generator Feeding Induction Motor” IEEE Trans on Energy Conversion, Vol. 9, Issue 2, June 1994 pp:390 -396 .

3. A. K. Al-Jabri, and A.L.; Alolah, "Capacitance Requirement For Isolated Self-Excited Induction Generator”. IEE Proc.Vol.137, pt. B, No.3, May 1990, pp. 154-159.

4. R.Bonert, S. Rajakaruna "Self-Excited Induction Generator With Excellent Voltage and Frequency Control” IEE Proc.-Gener.Transm.Distrib. ,Vol.145,No.1,Jan. 1998, pp. 33-39.

5. S.S.Murthy , G.Bhuvaneswari ,S.Gao ,M.S.Gayathri "Performance Analysis of a Self Excited Induction Generator With Digitally Controlled Electronic Load Controller for Micro Hydel Power Generation" Power System Technology and IEEE Power India Conf ,Oct. 2008, pp.1 - 6.

6. C.Grantham, D. Sutanto, and B.Mismail, "Steady State and Transient Analysis of Self-Excited Induction Generators”. IEE Proc Vol. 136, Pt. B, No.2, March 1989, pp. 61-68.

7. L.Shridhar, B.Singh, C.S.Jha "Self Regulation in Capacitor Excited Induction Generator" IEEE Electrical Machines and Drives Conf ,Sep.1993, pp.190 - 195.

8. E.C.Quispe , R.D.Arias, J.E.Quintero "A New Voltage Regulator for Self-Excited Induction Generator-Design, Simulation and experimental results" IEEE Electrical Machines and Drives Conf , May 1997, pp.TB3/7.1 - TB3/7.3 .

9. M.A. Al-Saffar, Eui-Cheol Nho, T.A.Lipo "Controlled Shunt Capacitor Self-Excited Induction Generator" IEEE Industry Applications Conf, Vol. 2, Oct. 1998, pp.1486 - 1490.

10. Basil M. Said "Self-Excited Induction Generator With Microcomp- uter-controlled PWM Inverter" Mutah Lil-Buhuth wad-Dirasat, Vol.15, No.4,2000, pp. 53-70.

11. M.E.Fraser, C.D.Maning "Performance of Average Current Mode Controllrd PWM UPS Inverter With High Crest Factor Load"IEEE Power Electronics and Variable-Speed Drives Conf No.399,Oct 1994, pp.661-667.

12. M. H. Rashid ,"Power Electronics Circuits, Devices and Applications", Third Edition, Electrical and Engineering, University of West Florida, United State of America, ISBN 0-13-122815-3, 2004. 


\section{ملحق )}

LS

1_ قيم ومقننات مسوق مغير التردد (Variable Frequency Drive).

SV 055 is5 , $7.5 \mathrm{hp} / 5.5 \mathrm{Kw}$.

Input (380-460) Volt $3 \Phi, 50 \mathrm{~Hz}, 16.8 \mathrm{~A}$.

Output $\quad 0 \longrightarrow$ input $3 \Phi, 12 \mathrm{~A}$. 2_ قيم عناصر الماكنة الحثية ثلاثية الطور و المحسوبة من فحصي الدائرة المفتوحة ودائرة غلق الدوار.

$\mathrm{Rs}=3.48 \mathrm{ohm}, \mathrm{Ls}=13.06 \mathrm{mH}$

$\mathrm{Rr}^{\prime}=2.72 \mathrm{ohm}, \mathrm{Lr}{ }^{\prime}=10.18 \mathrm{mH}$.

$\mathrm{Rm}=1907.7$ ohm. , Lm= $199.7 \mathrm{mH}$.

$$
\text { تم اجراء البحث في كلية ألهندة = جامعة ألموصل }
$$

